\title{
Olfactory Trace Conditioning in Drosophila
}

\author{
Dana Shani Galili, ${ }^{1}$ Alja Lüdke, ${ }^{2}$ C. Giovanni Galizia, ${ }^{2}$ Paul Szyszka, ${ }^{2}$ and Hiromu Tanimoto ${ }^{1}$ \\ ${ }^{1}$ Max-Planck Institut für Neurobiologie, 82152 Martinsried, Germany, and ${ }^{2}$ University of Konstanz, Department of Biology, Neurobiology, 78457 Konstanz, \\ Germany
}

The neural representation of a sensory stimulus evolves with time, and animals keep that representation even after stimulus cessation (i.e., a stimulus "trace"). To contrast the memories of an odor and an odor trace, we here establish a rigorous trace conditioning paradigm in the fruit fly, Drosophila melanogaster. We modify the olfactory associative learning paradigm, in which the odor and electric shock are presented with a temporal overlap (delay conditioning). Given a few-second temporal gap between the presentations of the odor and the shock in trace conditioning, the odor trace must be kept until the arrival of electric shock to form associative memory. We found that memories after trace and delay conditioning have striking similarities: both reached the same asymptotic learning level, although at different rates, and both kinds of memory have similar decay kinetics and highly correlated generalization profiles across odors. In search of the physiological correlate of the odor trace, we used in vivo calcium imaging to characterize the odor-evoked activity of the olfactory receptor neurons in the antennal lobe. After the offset of odor presentation, the receptor neurons showed persistent, odor-specific response patterns that lasted for a few seconds and were fundamentally different from the response patterns during the stimulation. Weak correlation between the behavioral odor generalization profile in trace conditioning and the physiological odor similarity profiles in the antennal lobe suggest that the odor trace used for associative learning may be encoded downstream of the olfactory receptor neurons.

\section{Introduction}

Animals can keep representations of sensory stimuli for a short while even after their cessation. Classical conditioning can be used to behaviorally reveal such stimulus traces (Pavlov, 1911). In trace conditioning, a conditioned stimulus (CS) and an unconditioned stimulus (US) are separated by a temporal gap (see Fig. 1). Therefore, a CS trace must be kept during the gap for the animal to learn the CS-US contingency, in contrast to delay conditioning, in which the CS and the US temporally overlap.

Trace conditioning has been reported in many different paradigms, such as eyeblink conditioning and fear conditioning in rodents and man (Solomon and Groccia-Ellison, 1996; McEchron et al., 1998; Woodruff-Pak and Disterhoft, 2008). In the mammalian nervous system, memories after delay and trace conditioning require mostly common neural pathways (Thompson and Krupa, 1994), whereas trace conditioning additionally recruits the hippocampus to bridge the temporal gap (Solomon et al., 1986; McEchron et al., 1998; Weiss et al., 1999; Woodruff-Pak and Disterhoft, 2008). Although basic associative circuitry involved in these paradigms is well described, little is

\footnotetext{
Received Dec. 17, 2010; revised Feb. 17, 2011; accepted March 2, 2011.

Author contributions: D.S.G., A.L., C.G.G., P.S., and H.T. designed research; D.S.G. and A.L. performed research; D.S.G., A.L., C.G.G., P.S., and H.T. analyzed data; D.S.G., A.L., P.S., and H.T. wrote the paper.

This work was supported by Bundesministerium für Bildung und Forschung Grant $01 G Q 0931$ (C.G.G., P.S., H.T.), Max-Planck Gesellschaft (H.T.), and Minerva Stiftung (D.S.G.). D.S.G. is a PhD student of International Max Planck Research School. We thank A. V. M. Herz, R. Jortner, G. Laurent, R. Menzel, A. Schaefer, and A. Yarali for inspiring discussions and for sharing ideas. We are also grateful for the excellent technical assistance by A. B. Friedrich, A. Gruschka, and I. Siwanowicz.

Correspondence should be addressed to Hiromu Tanimoto, Max-Planck Institut für Neurobiologie, Am Klopferspitz 18, 82152 Martinsried, Germany. E-mail: hiromut@neuro.mpg.de.

DOI:10.1523/JNEUROSCI.6667-10.2011

Copyright $\odot 2011$ the authors $\quad 0270-6474 / 11 / 317240-09 \$ 15.00 / 0$
}

understood about how CS representations per se evolve over time and how the neural correlate of the stimulus trace is generated (Thompson and Krupa, 1994; Bathellier et al., 2008). Given excellent experimental accessibility to the nervous system, insects may be an attractive model to study both behavioral and physiological properties of CS traces.

Insects can extract sensory information from stimulus traces. For example, Drosophila melanogaster can remember the position of a vanished visual object and use this information for navigation (Neuser et al., 2008). Moths form olfactory associative memory more effectively in trace rather than delay conditioning (Ito et al., 2008). Aversive olfactory learning in Drosophila has been applied to study the temporal dynamics of stimulus representations (Tully and Quinn, 1985; Tanimoto et al., 2004; Riemensperger et al., 2005; Tomchik and Davis, 2009), yet the residual odor after the removal was not explicitly controlled, which may be crucial to study odor traces.

In insects, an odorant is detected mainly by olfactory receptor neurons (ORNs) in the antenna and encoded by a specific combinatorial pattern of ORN activity (Galizia and Szyszka, 2008; Masse et al., 2009). In the antennal lobe, to which ORNs project, odor-evoked activity undergoes transformation through excitatory and inhibitory interactions (Wang et al., 2003; Wilson et al., 2004; Mazor and Laurent, 2005; Bhandawat et al., 2007; Olsen et al., 2007; Silbering and Galizia, 2007; Root et al., 2008; Masse et al., 2009). Projection neurons convey the processed odor signals to the lateral horn and the Kenyon cells of the mushroom body, in which the representation is further modified (Perez-Orive et al., 2002; Wang et al., 2004; Szyszka et al., 2005; Jortner et al., 2007; Masse et al., 2009). The odor information and the aversive reinforcement signal converge at the presynaptic terminals of the 


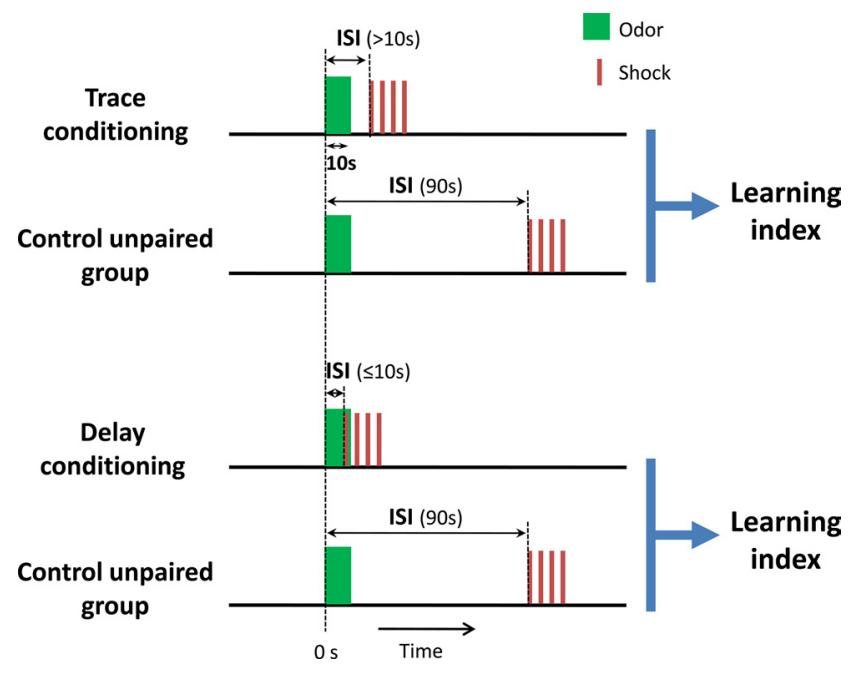

Figure 1. Experimental design of delay and trace conditioning. In single-odor conditioning, the two reciprocal groups of flies (conditioned and control unpaired groups) are used with an odor presentation for $10 \mathrm{~s}$. Time 0 indicates the odor onset. ISI for experimental groups is $\leq 10$ $s$ for delay and $>10 \mathrm{~s}$ for trace conditioning. The ISI for the corresponding control unpaired group is always $90 \mathrm{~s}$. The interval of multiple training trials is $210 \mathrm{~s}$. For calculating the learning index, first an avoidance index is calculated based on the choice of the flies in each reciprocally trained group. Then the $L \mathrm{l}$ is calculated as an average of the avoidance indices of the conditioned and the control unpaired groups (see Materials and Methods).

Kenyon cells to form aversive olfactory memory (Gerber et al., 2004; Davis, 2005). Although olfactory representations in insects are much investigated, little is understood on how the representations after stimulus offset are generated and used to form associative trace memory.

We here established a rigorous trace conditioning paradigm in Drosophila and compared odor memories in trace and delay conditioning. Furthermore, we combined behavioral and physiological approaches to search for the neuronal correlate of the perceived odor trace.

\section{Materials and Methods}

Behavioral assay. The wild-type D. melanogaster strain Canton-S was used for olfactory conditioning paradigms. Flies were reared on standard cornmeal medium at $25^{\circ} \mathrm{C}$ and $60 \%$ relative humidity. All flies were handled without anesthesia until experiments were commenced. Mixtures of male and female flies aged 2-6 d after eclosion were used for behavioral experiments.

A standard differential conditioning with two odorants (Tully and Quinn, 1985; Schwaerzel et al., 2002) was modified for single-odor learning (Figs. 1, $2 \mathrm{Ai}$ ). An odor was presented for $10 \mathrm{~s}$ followed by four electric shock pulses (each $1.25 \mathrm{~s}$ long at $90 \mathrm{~V}$, delivered every $5 \mathrm{~s}$ ) (Fig. 1). Interstimulus interval (ISI) was defined as the time between the odor onset and the shock onset (Fig. 1). Given the 10 s odor presentation, ISI $\leq 10 \mathrm{~s}$ was operationally defined as delay conditioning, whereas trace conditioning used an ISI $>10 \mathrm{~s}$ (Fig. 1). In parallel, the control unpaired group was presented with the same amount of odor and shock but with an ISI of $90 \mathrm{~s}$, which ensured no detectable associative learning (see Fig. 3A) (Tanimoto et al., 2004). Intertrial interval (i.e., the time between the first shock pulses in two consecutive trials) was set to $210 \mathrm{~s}$, when the training cycle was repeated.

Conditioned odor avoidance was measured immediately after the training (unless otherwise mentioned) in a T-maze (Tully and Quinn, 1985; Schwaerzel et al., 2002), where flies chose between the odor and a solvent [paraffin oil for 4-methylcyclohexanol ( $\mathrm{MCH})$, distilled water for all other odors]. The flies were given $2 \mathrm{~min}$ to distribute between the two arms of the T-maze. The number of flies in each arm was then counted to calculate their odor avoidance. An avoidance index was calculated as the difference between the number of flies distributed in each of the two arms, divided by the total number of flies. A learning index (LI) was then calculated as the mean avoidance of the paired and unpaired groups (Tully and Quinn, 1985; Yarali et al., 2009). For the generalization experiments (see Fig. $5 A, B$ ), flies were trained with 1-butanol and tested with another (new) odor. A generalization index was calculated by dividing the learning index of the tested odor by that of 1-butanol.

To control for a residual odorant in the training tube after the cessation of an odor presentation, we first scented an empty training tube with a given odor for $10 \mathrm{~s}$. Flies were introduced into the tube only after the removal of the odor followed by a $5 \mathrm{~s}$ air flush, and subsequently received electric shocks (Fig. 2Aii, Trace control). If the flies showed conditioned avoidance comparable with the "real" trace conditioning with ISI of $15 \mathrm{~s}$, the residual odor must have been sufficient to support learning. We tested five hydrophilic odorants (i.e., 1-butanol diluted 1:50; acetic acid diluted 1:20; 1-propanol diluted 1:50; propionic acid diluted 1:20; and ethyl acetate diluted 1:20) and one hydrophobic odorant, MCH (1:100) (Fig. 2B). Odor concentrations were calibrated to induce similar memory in delay conditioning (Fig. $2 B$ ).

Data analysis: behavior. The significance level of statistical tests was set to 0.05 . None of the groups tested in associative learning violated the assumptions of the normal distribution and the homogeneity of variance. Therefore, parametric tests were used: one- or two-sample $t$ test, one- or two-way ANOVA followed by multiple comparisons with Bonferroni-Holm correction, or Pearson's correlation coefficient. Because the distribution of the avoidance indices was significantly different from the normal distribution (see Fig. $3 A$ ), the medians of the groups were compared with the nonparametric Kruskal-Wallis test. All statistical analyses were made using the software Prism 5 (GraphPad Software). The sample size of each group and the results of each test are reported in the corresponding figure legends. Unless otherwise stated, bars and error bars represent means and SEM throughout the paper.

In vivo calcium imaging. Odor responses in ORNs were recorded in the genotype $y$ w UAS-G-CaMP1.3; Or83b-Gal4. These flies express a fluorescence sensor G-CaMP (Nakai et al., 2001) in $\sim 60 \%$ of all ORNs (Larsson et al., 2004).

For imaging, adult female flies (aged 7-14 d) were prepared as described previously (Silbering and Galizia, 2007). The antennal lobe was imaged with a fluorescence microscope (BX-50 WI; Olympus) equipped with a water-dip objective (40×, numerical aperture 1.0; Carl Zeiss) and a CCD camera (Imago QE; T.I.L.L. Photonics). Excitation light of 475 $\mathrm{nm}$, which was generated with a monochromator (Polychrome II; T.I.L.L. Photonics), was filtered by a $500 \mathrm{~nm}$ short-pass filter. Excitation and emission light were separated by a $495 \mathrm{~nm}$ dichroic mirror and a 505 $\mathrm{nm}$ long-pass filter. Exposure time was $180 \mathrm{~ms}$. To adjust different basal fluorescence values, gray filters $(5,10$, and $32 \%$ transmission) were inserted into the excitation light beam. Four by four pixels of the camera were binned on-chip, resulting in a resolution of $160 \times 120$ pixels, corresponding to $145 \times 109 \mu \mathrm{m}$ on the preparation. We recorded with an acquisition rate of $5 \mathrm{~Hz}$ for $35 \mathrm{~s}$ ( 175 frames), and odors were applied for $10 \mathrm{~s}$ in a pseudorandomized order. The interval between measurement onsets was $2 \mathrm{~min}$. Odor stimulation was controlled by the acquisition software of the imaging system (T.I.L.L. Vision; T.I.L.L. Photonics).

Odors (Sigma-Aldrich) were diluted in mineral oil (Sigma-Aldrich) to distinct final concentrations, which were 10 times lower than the concentrations for the behavioral experiments as a result of the direct odor application method via an olfactometer. The diluted odors were stored in $100 \mathrm{ml}$ rolled-flange glass bottles (Thermo Fisher Scientific), sealed with silicon-Teflon septa (Schmidlin Labor Service). The bottles were connected to a custom-built, computer-controlled olfactometer (companion paper by Szyszka et al., 2011) via needles (1.2 $\mathrm{mm}$ diameter) through the septum. New odors were prepared every 4 weeks. A constant airstream $(3 \mathrm{~L} / \mathrm{min})$ was applied to the fly's antennae through a glass tube (inner diameter of $6.2 \mathrm{~mm}$ ), which was located $\sim 8 \mathrm{~mm}$ away from the fly. This constant airstream was the sum of a carrier airstream $(1.2 \mathrm{~L} / \mathrm{min})$ and six channels (each $0.3 \mathrm{~L} / \mathrm{min}$ ). The olfactometer produced nearly rectangular odor pulses with steep odor onsets and offsets, as measured using a photoionization detector (model 200a; Aurora Scientific). Continuous air suction behind the fly cleared residual odor.

Data analysis: imaging. The imaging data were analyzed with custommade routines written in IDL (Research Systems Inc.) and R (www.r- 
project.org). Only flies that responded throughout the experiment with stable calcium signals were included in data analysis. First, lateral movement was corrected by means of anatomical landmarks within and between measurements. Glomeruli were identified based on anatomical cues and on their response profiles (according to Silbering and Galizia, 2007; Silbering et al., 2008). The glomerular response patterns for 1-butanol and propionic acid corresponded well to the previously described response patterns (Silbering et al., 2008).

Glomerular responses were calculated by averaging the light intensity of $7 \times 7$ pixels in the glomerulus center (corresponding to $6.3 \times 6.3$ $\mu \mathrm{m})$. The relative change in fluorescence was calculated for each frame $i$, as $(\Delta F / F)_{i}(\%)=$ $\left(F(i)-F_{0}\right) / F_{0} \times 100 . F(i)$ is the absolute fluorescence of the $i$ th frame, and $F_{0}$ is the background fluorescence, which was calculated as the average fluorescence of 15 frames before odor stimulation (frames 10-25). A bleach correction was applied to the signals by fitting a logarithmic function $\left(F(t)=a \times e^{(b t)}+c\right)$ to the average light intensity change in each glomerulus over time (Galizia and Vetter, 2004). The frames $25-155$, covering 26 s after stimulus onset, were excluded from the curve fitting, to make the investigation of odor and postodor responses possible. The frames before stimulation (frames 3-24) were weighted twofold in the correction because, at the beginning of the measurements, the bleaching was the strongest. For few glomerular signals the curve fitting with these parameters was not sufficient; thus, they were changed slightly [frames $4-24$ were weighted onefold, and frames 25-172 (= $29.4 \mathrm{~s}$ ) were excluded]. To quantify the similarity between pairs of odor responses over time (see Figs. 4, 5), odor responses were first calculated as vectors that contained the signal values of all glomeruli recorded in all flies. Then, the similarity between response patterns was quantified by a sliding window Pearson's correlation which can range from -1 (anticorrelated) to 1 (perfectly correlated).

\section{Results}

We compared two forms of olfactory classical conditioning: trace and delay conditioning, which differ only in the ISI (the time from the CS onset to the US onset). In trace conditioning the odor presentation ceases before the onset of electric shock punishment, whereas it ceases after the onset of the electric shock in delay conditioning (Fig. 1). We modified the commonly used conditioning design by presenting a single odor for $10 \mathrm{~s}$ followed by four pulses of electric shock to confer temporal specificity and compatibility with our physiological measurements (Fig. 1). Thus, ISI for delay conditioning was $\leq 10 \mathrm{~s}$, and ISI for trace conditioning was $>10 \mathrm{~s}$.

\section{Establishing a reliable paradigm for trace conditioning}

For delay (ISI $=10 \mathrm{~s}$ ) and trace (ISI $=15 \mathrm{~s}$ ) conditioning, we tested six different odorants. To minimize potential adhesion of odorants to the apparatus made of plastic, we chose five hydrophilic odorants (Fig. $2 \mathrm{~B}$ ) and $\mathrm{MCH}$, a commonly used hydrophobic odorant. The concentration of each odorant was adjusted to elicit comparable levels of memory in single-trial delay conditioning (Fig. $2 \mathrm{~B}$ ). Under these conditions, three odors induced significant memory after single-trial trace conditioning $(\mathrm{MCH}$, $p=0.019 ;$ propanol, $p=0.031 ;$ butanol, $p=0.003$; propionic acid, $p>0.05$; acetic acid, $p>0.05$; ethyl acetate, $p>0.05$ ) (Fig. 2 B)

As a prerequisite to study trace conditioning, it is crucial to ensure that animals learn the task based on a neuronal odor trace but not on residual odor lingering in the training apparatus after the cessation of the presentation. Because flies are able to associate very low odor concentrations with shock (Yarali et al., 2009), we developed a control experiment, in which an empty training tube was scented for $10 \mathrm{~s}$ before the introduction of flies and then flushed with air for $5 \mathrm{~s}$ as was done in trace conditioning (Fig. $2 A)$. The electric shock was applied only after the introduction of flies (Fig. 2 Aii, Trace control). We found that the memory of the trace control experiment was significantly lower than the memory of trace conditioning when 1-butanol was used $(p=0.014)$ (Fig. $2 B$ ). Thus, we used 1-butanol for all following experiments. In contrast, residual $\mathrm{MCH}$ after odor removal and air flush was sufficient to induce memory indistinguishable from the memory acquired during the trace conditioning procedure $(p=0.58)$ (Fig. $2 B$ ), suggesting that trace conditioning with $\mathrm{MCH}$ is confounded by lingering odor molecules.

\section{Dependence of conditioning on stimulus timing}

We next asked how long an odor trace can be kept available for associative memory formation. We trained flies twice with various ISIs ranging from 5 to $30 \mathrm{~s}$. The conditioned ("paired") groups avoided the odor differently depending on the ISI $p<$ 0.001 , Kruskal-Wallis test). The avoidance index of pooled recipro- 
A

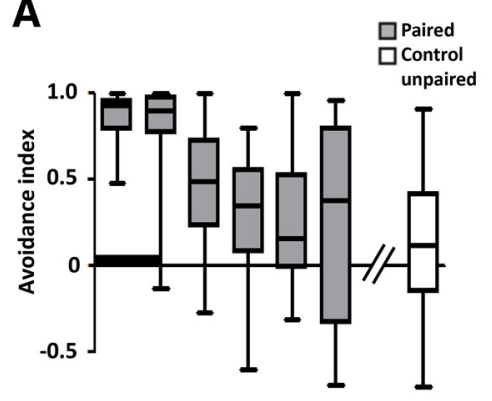

$\begin{array}{llllll}5 & 10 & 15 & 20 & 25 & 30\end{array}$

ISI (s)
B

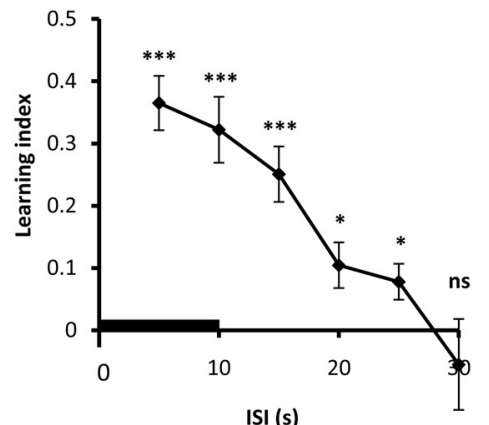

C

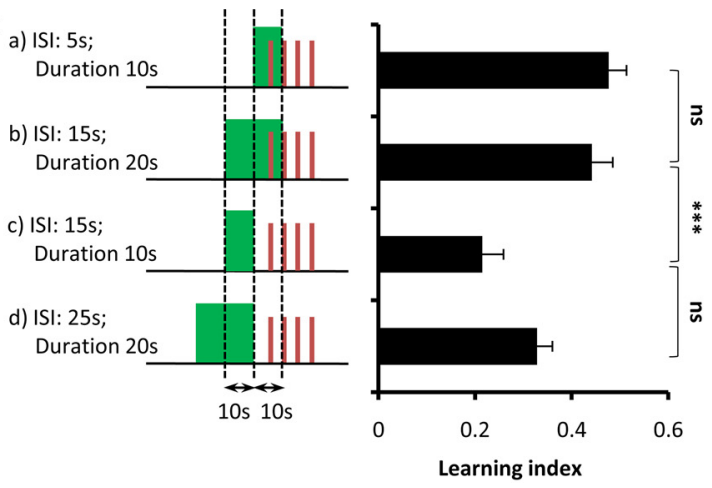

D

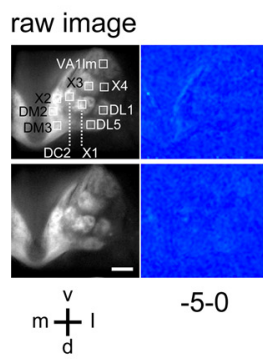

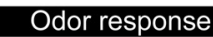

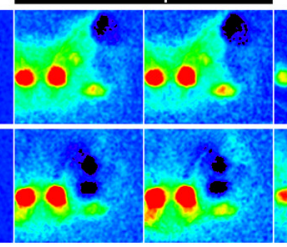

$0-5$
5-10

Time after odor onset (s)

10-15
Post-odor response
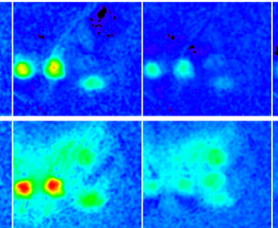

$15-20$

$20-25$

$25-30$
E

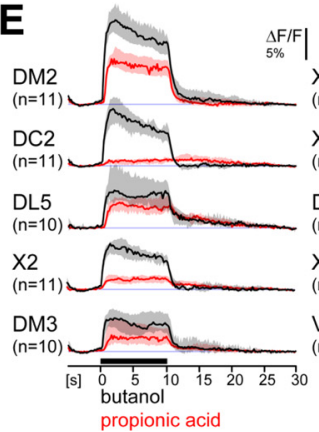

Figure 3. Odor traces in behavior and physiology. A, Avoidance index of the paired (gray) and control unpaired (white) groups after two trials is plotted as a function of ISI. ISI ranged from 5 to 30 s. A 10 s butanol stimulus is used as the (S presentation (black bar). Bars, boxes, and whiskers indicate medians, interquartiles, and $10-90$ percentiles, respectively. The control unpaired groups are pooled because the ISI is constant $(90 \mathrm{~s})$ and the avoidance indices are not significantly different $p>0.05$, Kruskal-Wallis test). The median avoidance indices of the paired groups were significantly different $\left(p<0.001\right.$, Kruskal-Wallis test). $\boldsymbol{B}$, Learning indices as a function of ISI. Memory levels are significantly different from zero for ISIs between 5 and $25 \mathrm{~s}$ (ISI $=5 \mathrm{~s}, t_{(19)}=8.3$, $\left.p<0.001 ;|I S|=10 s, t_{(17)}=6.07, p<0.001 ; I S I=15 s, t_{(19)}=5.6, p<0.001 ;\left|S I=20 s, t_{(19)}=2.8, p=0.03 ;\right| S\left|=25 s, t_{(18)}=2.6, p=0.03 ;\right| I S I=30 s, t_{(17)}=0.7, p=0.46\right) . C, A$ temporal gap between the odor offset and the shock onset is critical for trace learning. Four groups are different in terms of odor presentations (i.e., ISI and odor duration): group a, ISI $=5 \mathrm{~s}$, duration $=10 \mathrm{~s}$; group b, ISI $=15 \mathrm{~s}$, duration $=20 \mathrm{~s}$; group $c, I S I=15 \mathrm{~s}$ duration $=10 \mathrm{~s}$; group d, ISI $=25 \mathrm{~s}$, duration $=20 \mathrm{~s}$. The learning indices of the four groups are significantly different $\left(F_{(3,97)}=9.0, p<\right.$ 0.001 , one-way ANOVA; $t_{\text {(b v c })}=4.09, p=0.003 ; t_{\text {(a v s })}=0.63, p=0.54 ; t_{\text {(c vs d) }}=2.04, p=0.08 ; t_{\text {(a vs c) }}=4.67 p<0.001$ ). $n=18-26 . D$, (alcium responses of 0RNs in the antennal lobe using Or83b-GAL4/UAS-G-CaMP1.3. The raw image on the left shows the G-CaMP fluorescence of the right antennal lobe. Color-coded images show the fluorescence changes (averaged over $5 \mathrm{~s}$ windows) during and after stimulation with butanol or propionic acid. Scale bar, $20 \mu \mathrm{m}$. $E$, Time course of ORN responses to butanol (black) and propionic acid (red) in individual glomeruli (median of responses from 9 to 12 flies; shaded area indicates the 25 and $75 \%$ quartiles). Odor stimuli induce positive or negative signals (odor response) often followed by positive signals that last for several seconds (post-odor response). For glomeruli X1-X4, an unambiguous identification was not possible. ${ }^{*} p<0.05 ;{ }^{* *} p<0.01 ;{ }^{* * *} p<0.001$.

cal unpaired groups ("control unpaired"; ISI = $90 \mathrm{~s}$ ) was approximately zero (Fig. $3 A$ ). Accordingly, learning indices progressively decreased with increasing ISI (Fig. $3 B$ ). These results indicate that flies can keep the trace of a $10 \mathrm{~s}$ odor presentation for at least $15 \mathrm{~s}$ after the offset and suggest that the transition between delay and trace memories may be continuous.

Next, we addressed whether the onset or the late phase/offset of the odor stimulus signals the representation for trace memory. By modifying odor duration and ISI, we compared the memories of groups trained with the same odor onset time but different offset times or with the same odor offset time but different onset times (Fig. 3C). Odor presentations with the same ISIs (i.e., same odor onset) but different durations (i.e., different odor offset) produced significantly different memory levels (Fig. 3C): the odor presentation that temporally overlapped with the shock (delay) was more effective than the odor presentation with the same ISI but without a temporal overlap (trace) (Fig. 3C, groups $\mathrm{b}$ vs $\mathrm{c}, p=0.003$ ). Changing the odor duration and onset (i.e., ISI) while maintaining the same interval time between odor offset and shock onset did not significantly affect memory (Fig. $3 C$, groups a vs $b, p>0.05$; groups $c$ vs $d, p>0.05$ ). These results suggest that the stimulus-free interval between the odor offset and the shock onset is critical for the decreased memory observed in trace conditioning.

\section{Spatiotemporal pattern of odor representations in the antennal lobe}

We hypothesized that ORNs in the antennal lobe could encode the odor trace. We therefore measured calcium responses in ORNs during odor stimulation (odor response) and after odor offset (post-odor responses), by targeting G-CaMP expression using Or83b-GAL4 (Fig. 3D). Post-odor ORN responses in Drosophila have been found previously (Hallem et al., 2004; Silbering and Galizia, 2007; Silbering et al., 2008), but their specificity and dynamics have not been studied in detail. Odors were presented using the same design as in the behavioral experiments, as 10-s-long pulses. Each odor induced an odor-specific pattern of positive and negative glomerular responses as visualized in the color-coded signal images for a single fly (Fig. 3D) and in the time courses for each glomerulus averaged over several flies (Fig. 3E). After odor offset, the response pattern changed into a post-odor response pattern, which was odor and glomerulus specific and lasted for $\sim 10 \mathrm{~s}$. This post-odor response could not be predicted from the odor response pattern, and the direction of response change depended on the glomerulus/odor combination. Glomerulus DC2, for example, showed a strong odor response to butanol, which went back to baseline within $2 \mathrm{~s}$ after offset. In contrast, the response of DC2 to propionic acid was weak during the odor presentation, but its post-odor response was sustained for $\sim 10 \mathrm{~s}$ after odor 
offset (Fig. 3E). In glomerulus X3, butanol evoked a positive odor response, whereas propionic acid evoked a negative odor response followed by a positive post-odor response, which lasted for $\sim 10 \mathrm{~s}$ (Fig. $3 E$ ).

\section{Post-odor responses in ORNs are reproducible and odor specific}

We next analyzed the dynamics and specificity of the ORN odor and post-odor responses, with a time-resolved quantification of their pattern similarity within and between measurements (Fig. 4). Analyses are based on combined responses from nine flies. Each pixel in the similarity matrices represents the correlation between two response patterns at given time points. The similarity matrix in Figure $4 \mathrm{~A}$ shows the pattern similarity within (quadrants I and III) and between (quadrants II and IV) two measurements of $10 \mathrm{~s}$ butanol stimulation. Before stimulation, the correlation fluctuated around 0 (low similarity in area b in Fig. 4A). During odor presentation, the pattern was stable for the entire stimulation time of $10 \mathrm{~s}$ (increased similarity in area c). The subsequent post-odor response was also stable for $\sim 10 \mathrm{~s}$ after odor offset (high similarity in area d) but was different from the odor response (low similarity in area e). Both odor response and post-odor response pattern were reproducible (high similarity in areas $\mathrm{f}$ and $\mathrm{g}$ ). The matrix in Figure $4 B$ shows the similarity within and between response patterns to butanol and propionic acid. Likewise, the pattern similarity with all the other odors was high within the odor or post-odor response but low between odor and post-odor responses (data not shown). The pattern similarity was lower across odors than within-odor similarity for both odor and post-odor responses [in Fig. 4B, compare cross-odor similarity between butanol and propionic acid (quadrants II and IV) and butanol (quadrant I) or propionic acid (quadrant III)].

We next quantified the dynamic of changes in pattern similarity of the butanol and post-butanol responses, by comparing the activity pattern at a single timepoint with the activity patterns at all time points (Fig. $4 C$ ). The initial butanol response pattern turned dissimilar within $3 \mathrm{~s}$ after odor offset, and its similarity between the initial and post-odor responses fluctuated around 0 (Fig. $4 C$, magenta trace; similarity values from area $\mathrm{h}$ in Fig. $4 \mathrm{~A}$ ). The post-butanol response pattern showed increased similarity immediately after odor offset and remained stable for $\sim 10 \mathrm{~s}$ (Fig. $4 \mathrm{C}$, green trace; similarity values from area $\mathrm{i}$ in Fig. 4A). Both the butanol and the post-butanol response patterns were more similar between repeated stimulations than between butanol and different odors (Fig. 4D), indicating that odor and post-odor response patterns were odor specific. This raises the question: does either of these odor representations serve for associative memory in trace conditioning? If it did, the degree of
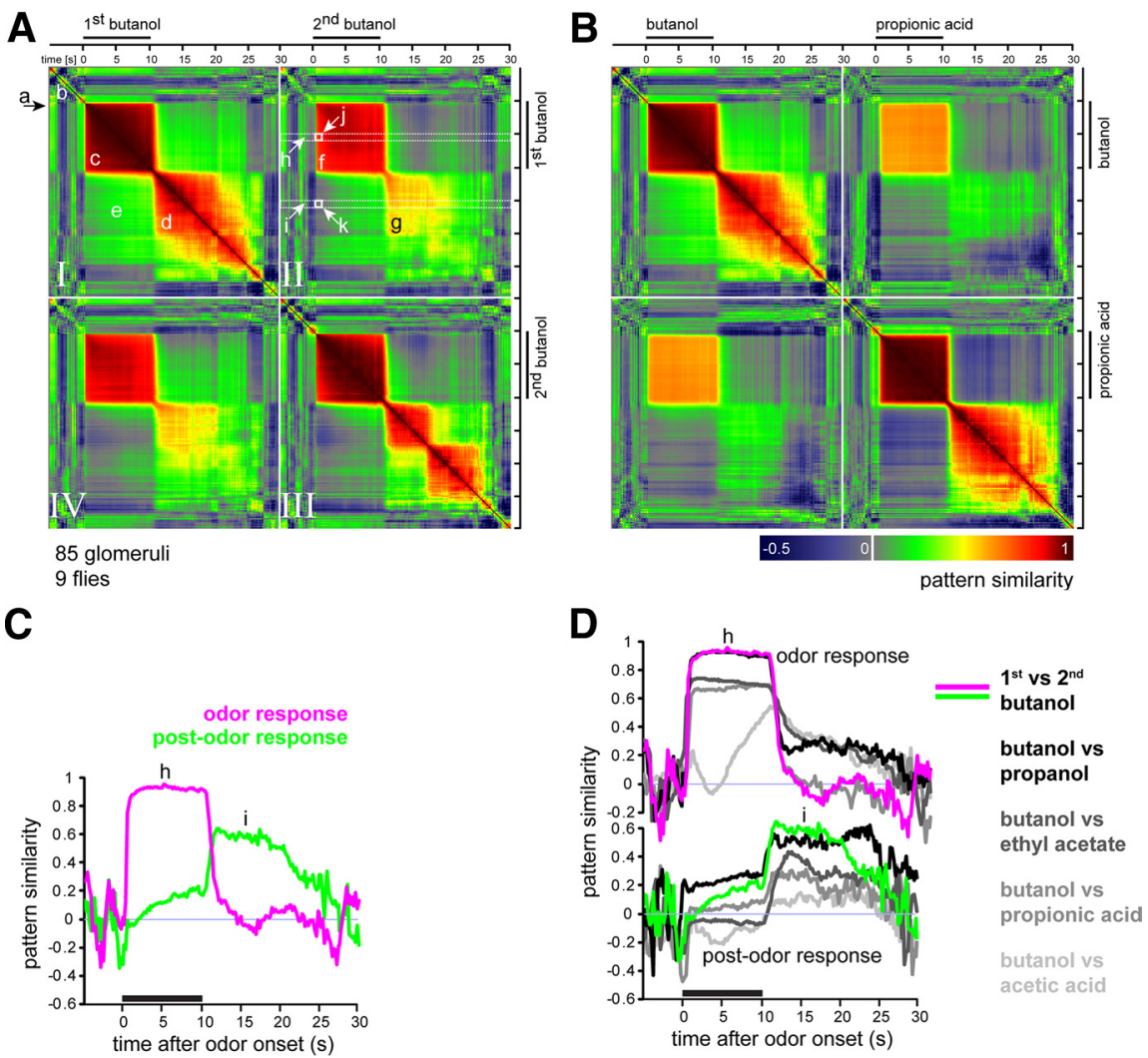

Figure 4. Post-odor responses in ORNs are reproducible and odor specific. $\boldsymbol{A}$, The similarity matrix shows the time-resolved similarity between response patterns within (quadrants I and III) and between (quadrants II and IV) measurements. Response patterns are calculated as vectors with signal values of 85 glomeruli recorded in nine flies. Similarity between these response 作 ( quantified within and between butanol and propionic acid measurements. For both odor and post-odor responses, the similarity between butanol and propionic acid (quadrant II) is lower than within butanol (quadrant I) or within propionic acid (quadrant III). C, Magenta trace, Time-resolved similarity between the odor response pattern (5-6 s after odor onset) of the first butanol measurement and the response patterns at all time points of the second butanol measurement (similarity values from $h$ in $\boldsymbol{A}$ ). trace, Time-resolved similarity between the post-odor response pattern (15-16 s after odor onset) of the first butanol measurement and the response patterns at all time points of the second butanol measurement (similarity values from i in $\boldsymbol{A}$ ). $\boldsymbol{D}$, Time-resolved similarity between the odor and post-odor response pattern of the first butanol measurement and the response races same as in $\mathbf{C}$ ). Both odor responses and post-odor responses are odor specific, because the similarity is higher between repeated butanol measurements than between butanol and the different odors ( $n=85$ glomeruli, 9 flies).

odor similarity in ORN responses should predict the degree of odor similarity in trace conditioning.

\section{Odor similarity in delay and trace conditioning}

To compare the perceived odor similarities in trace and delay memories, we used generalization learning (Guerrieri et al., 2005; Mishra et al., 2010). Flies were trained with two trials of either delay (ISI $=5 \mathrm{~s}$ ) or trace (ISI $=15 \mathrm{~s}$ ) conditioning with 1-butanol. Subsequently, they were tested for the avoidance of one of four different odors, which was new to the flies (Fig. 5A). Conditioned avoidance of the test odor depends on the perceived similarity between 1-butanol and the new test odor. Tests with the new odors revealed a different degree of odor generalization in trace conditioning, indicating that trace memory is odor specific (Fig. 5A). The odor generalization profiles following delay 

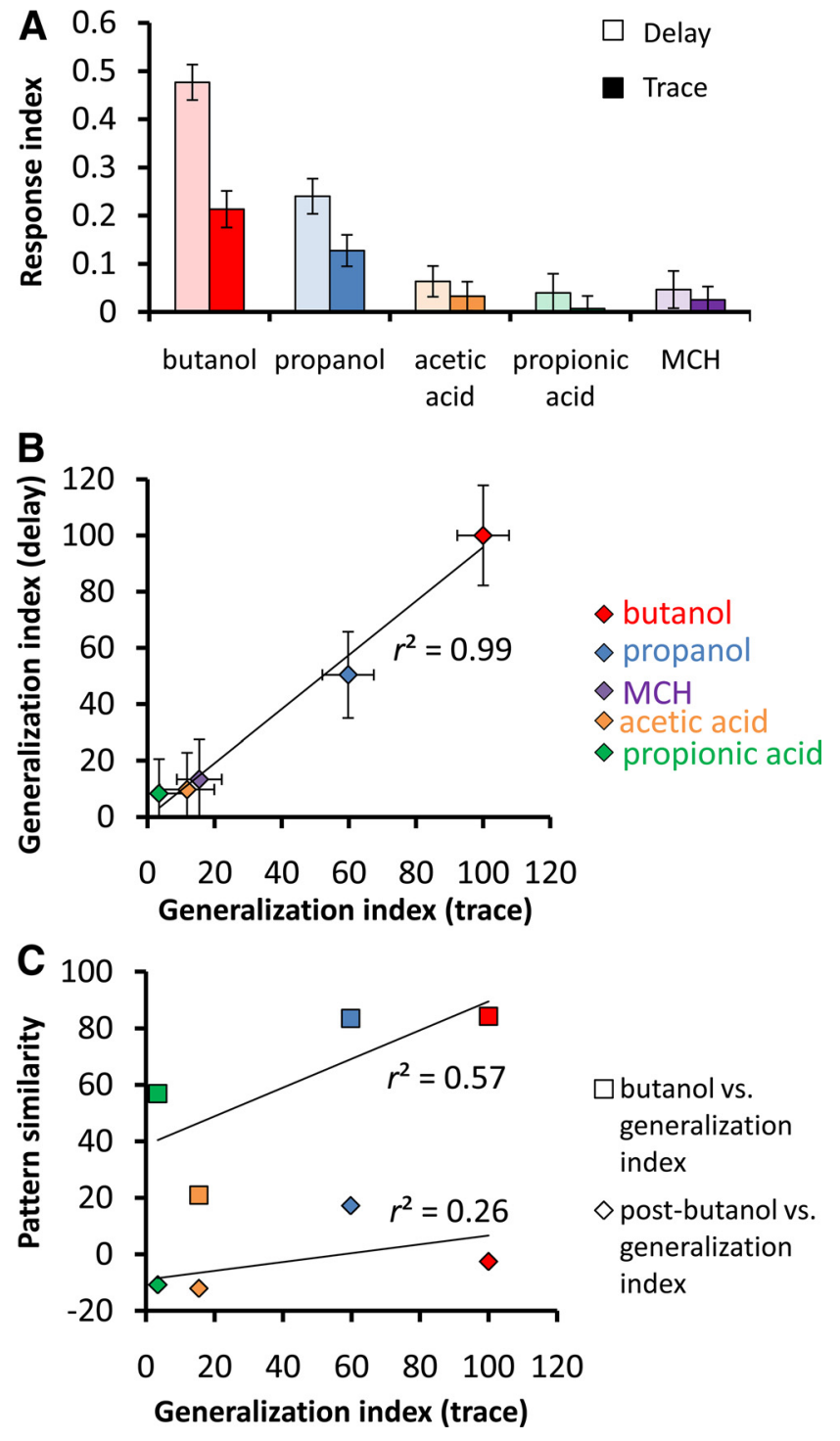

Figure 5. Perceived odor similarity in delay and trace conditioning. $\boldsymbol{A}, 0$ dor generalization for delay and trace conditioning. All groups received two trials of training with butanol as in delay (ISI = 5s) or trace conditioning (ISI = $15 \mathrm{~s}$ ) and tested for conditioned odor avoidance with a given odor (butanol or a novel odor). $\boldsymbol{B}$, Correlation between generalization indices for delay and trace conditioning. Test odors are color coded as in $\boldsymbol{A}$. Generalization profiles correlated highly (Pearson's $r^{2}=0.99, p<0.001$ ). C, Comparison of the behavioral generalization profile after trace conditioning with butanol (same data as in $\boldsymbol{B}$ ) and the similarity profile for the respective physiological ORN response patterns (same data as in Fig. 4). Dot plot shows behavioral generalization indices versus physiological pattern similarity between the butanol and the initial test odor responses. Square points, Generalization indices versus ORN similarity profile between the butanol response ( $5-6 \mathrm{~s}$ after odor onset) and the initial response of a given odor $(0-1 \mathrm{~s}$ after the odor onset, averaged values in box $j$ in Fig. $4 A)$. Diamonds, Generalization indices versus $\mathrm{ORN}$ similarity profile between the post-butanol response (15-16 $\mathrm{s}$ after odor onset) and the initial response of a given odor ( $0-1 \mathrm{~s}$ after the odor onset, averaged values in box k in Fig. $4 \mathrm{~A}$ ). The behavioral generalization profile barely correlates with the pattern similarity profiles of the butanol $\left(r^{2}=0.57\right)$ and the post-butanol responses $\left(r^{2}=0.26\right)$.

and trace conditioning were highly correlated (Fig. $5 A, B, r^{2}=$ 0.99 ), suggesting that the odor specificity in trace conditioning is comparable with that in delay conditioning. These results imply that the perception of butanol in delay and trace conditioning is similar to account for this high correlation. We further compared the generalization profile after trace conditioning with the similarity profiles of odor and post-odor response patterns of ORNs. We found that the behavioral generalization profile is not pre- dicted better by similarity profiles for the post-odor response than by the similarity profiles for the odor response (Fig. 5C). Thus, the neuronal odor trace that is associated with the shock may be generated downstream of the ORNs.

\section{Memories of delay and trace conditioning have similar characteristics}

The high similarity between delay and trace generalization profiles (Fig. 5B) prompted us to further examine the commonalities between the two types of memory. We compared memory acquisition and decay. As for acquisition, both delay and trace memories increased by repeating training trials (Fig. 6A). A single trial of trace conditioning was sufficient to produce significant memory (Figs. $2 B, 6 A$ ), although delay memory was significantly higher after one-, two-, or four-cycle training (one trial, $p<0.01$; two trials, $p<0.001$; four trials, $p=0.016$; eight trials, $p=0.76$; 12 trials $p=0.9$ ) (Fig. 6A). Interestingly, trace and delay memories reached the same asymptotic levels if the training trials were repeated sufficiently (Fig. 6A). This result implies that the perceived odor identity in delay and trace conditioning may be similar. Additionally, the different memory acquisition rates and a similar asymptote might reflect a difference in odor saliency between delay and trace conditioning, given the assumption of a theoretical model for learning acquisition by Rescorla and Wagner (1972).

We proceeded to measure how these similar short-term memories of trace and delay conditioning decay. Flies received eight training trials in delay or trace conditioning to achieve similar initial memory levels, and memory retention was tested at distinct time points up to $24 \mathrm{~h}$. We found very similar decay kinetics between delay and trace conditioning $\left(\tau_{\text {delay }}=6.8 \mathrm{~h}\right.$ and $\tau_{\text {trace }}=$ $6.3 \mathrm{~h}$ in a one-phase exponential decay) (Fig. $6 \mathrm{~B}$ ); delay and trace memories at any time point were not significantly different.

\section{Former association improves trace conditioning}

The memory score in trace conditioning was lower with few training trials, but it became indistinguishable from delay memory after repeated training (Fig. 6A). We hypothesized that previous associative training can help bridge the temporal gap in trace conditioning. To test this hypothesis, we trained two groups of flies with varying ISIs ranging from 15 to $35 \mathrm{~s}$ (Fig. 7A). Both groups received five trials of trace conditioning, and the only difference between these two groups was the trial sequence; one group received training with ascending ISIs and the other with descending ISIs. Interestingly, the memory of the ascending group was significantly higher than the memory of the descending group $(p=0.01)$ (Fig. $7 B$ ). Thus, former trace conditioning improves the ability to bridge the longer gap between the odor and the shock that flies otherwise do not learn well (Fig. 3A).

\section{Discussion}

This study, for the first time, compares olfactory delay and trace conditioning in D. melanogaster. Delay and trace memories share striking commonalities. Odor generalization profiles of delay and trace are highly similar (Fig. 5A,B). Trace and delay memories reach the same asymptote and have comparable decay kinetics (Fig. 6A,B). Together, these data suggest a similar perception of the odor identity and similar mechanisms for memory formation in delay and trace conditioning.

\section{Odor traces and trace memory in the fly}

We established a new paradigm to compare memories after trace and delay conditioning. Our study also features an important technical point, demonstrating that odorants can linger in the 
training apparatus (Fig. $2 \mathrm{~B}$ ). This residual odor after the offset appears to vary across different odorants, and notably, $\mathrm{MCH}$, a widely used odorant in Drosophila, was disapproved (Fig. 2 B). Hence, the butanol trace originates from the fly itself, not the apparatus, although the possibility of odorant adhesion on the cuticle or slow degradation in the lymph of the sensillum cannot be excluded.

Compared with delay conditioning, trace conditioning was less effective, although it reached the same asymptotic level after sufficient repetitions of training (Fig. 6A). Could flies perceive the odor trace as a less salient odor? According to the Rescorla-Wagner model, one of the robust models of learning acquisition (Rescorla and Wagner, 1972), CS saliency specifically changes the slope (i.e., rate), but not the asymptote, of learning acquisition. In insect olfactory learning, changing odor saliency was indeed reported to affect memory formation (Pelz et al., 1997; Masek and Heisenberg, 2008; Yarali et al., 2009). Together, our data suggest that the odor trace retains stimulus specificity but might be perceived less saliently than during the odor presentation.

\section{Dynamics of odor representations and odor traces}

Odor representations constantly change (Galán et al., 2006; Schaefer and Margrie, 2007). The spatiotemporal odor-evoked activity in the ORNs is regulated through the dynamics of ligandreceptor binding in the ORN and complex modulations by the antennal lobe network (for review, see Galizia and Szyszka, 2008, Masse et al., 2009). By imaging calcium signals of ORNs, we found long-lasting odor-evoked glomerular activity after the odor offset (Fig. 3C,D). This persistent post-odor activity was odor specific and different from the response pattern during the odor presentation (Fig. 4).

The specific poststimulus activity may be an evolutionarily conserved function of the olfactory system. The odor responses of both the honeybee projection neurons and the mouse mitral cells last long after the cessation of an odor presentation (Bathellier et al., 2008; companion paper by Szyszka et al., 2011). The glomerular patterns of the post-odor responses are odor specific and distinct from the patterns during the odor presentation (Galán et al., 2006; Bathellier et al., 2008; companion paper by Szyszka et al., 2011). Similarly, a small fraction of projection neurons in Drosophila also showed sustained firing activity after the odor stimulation (Bhandawat et al., 2007; Silbering and Galizia, 2007). However, the post-odor pattern similarity profiles in the antennal lobe did not predict the odor generalization profile in behavior (Fig. 5C) (companion paper by Szyszka et al., 2011). Altogether, these data suggest that (1) the representation of the odor trace may be maintained downstream in the olfactory system or (2) the odor traces may be represented by signals that are not reflected by elevated calcium concentrations. The significance of post-odor activity in the olfactory system remains to be clarified.

Along with projection neurons, Kenyon cells may keep the odor trace, because they play a major role in olfactory learning in insects and specifically in flies (Gerber et al., 2004; Davis, 2005). Trace conditioning in the moth, however, revealed that the odorevoked spiking activity of Kenyon cells is unlikely to be the representation used for the formation of associative memory (Ito et
A Ascending and descending ISI (s) in multiple training trials

\begin{tabular}{|l|l|l|l|l|l|}
\hline Troup & $\mathbf{1}$ & $\mathbf{2}$ & $\mathbf{3}$ & $\mathbf{4}$ & $\mathbf{5}$ \\
\hline Ascending & 15 & 20 & 25 & 30 & 35 \\
\hline Descending & 35 & 30 & 25 & 20 & 15 \\
\hline B & & & \\
\hline
\end{tabular}

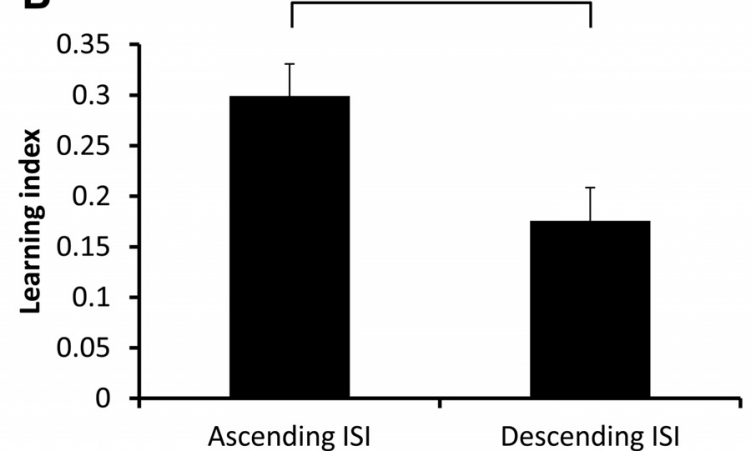

Figure 7. Former association improves trace conditioning. $\boldsymbol{A}$, Schematic description of the experimental design. Flies received five repeated trace training trials with different ISIs ranging from 15 to 35 s in either the ascending or descending manner. $\boldsymbol{B}$, Memory in the group with the ascending ISIs is significantly higher than in the group with the descending ISIs $\left(t_{(30)}=2.72\right.$; $p=0.01) \cdot n=16 .{ }^{*} p<0.05$.

al., 2008). As in the moth, there is little spiking activity of Drosophila Kenyon cells after the odor presentation (Turner et al., 2008). However, residual calcium after the odor presentation in either Kenyon cells or projection neurons may lower the threshold of reactivation during the presentation of the US. This may be a plausible scenario considering the prolongation of odor-evoked Kenyon cell activity in the presence of a sugar reward (Szyszka et al., 2008), and a new set of projection neurons recruited during associative training (Daly et al., 2004; Yu et al., 2004) with the presentation of a US. Thus, it would be important to measure post-odor neuronal activity during delay and trace conditioning also downstream in projection neurons and Kenyon cells (Daly et al., 2004; Yu et al., 2004; Szyszka et al., 2008).

Alternatively, the odor trace might be maintained as signals other than calcium, such as cAMP signaling (Bellmann et al., 2010). Therefore, measurements of the odor-evoked cAMP/pro- 
tein kinase A activity and modulations by associative learning would be important (Tomchik and Davis, 2009; Gervasi et al., 2010).

\section{Specific neural systems for trace memory?}

In mammals, it is widely accepted that trace conditioning recruits additional systems to those required for delay conditioning, such as the hippocampus (Solomon et al., 1986; Woodruff-Pak and Disterhoft, 2008) and state of awareness (Clark and Squire, 1998; Clark et al., 2002). Therefore, trace memory is proposed to be qualitatively different from delay memory, although other views have been suggested (LaBar and Disterhoft, 1998; Beylin et al., 2001; Carter et al., 2003; Kehoe et al., 2009). Our study in Drosophila revealed a behavioral continuum between delay and trace memories (Figs. 3A, 5, 6), but nonetheless, trace conditioning may recruit specific neural circuitry, which might be detectable only with specific intervention.

We found that trace conditioning with shorter ISIs enables the bridging of later odor-shock pairing with longer ISIs (Fig. 7). In bees, this facilitation specifically depends on the experience of a previous trace but not delay conditioning trial (companion paper by Szyszka et al., 2011). What could be the underlying mechanism of the facilitation? It was shown that, after associative conditioning, insects learn to anticipate upcoming US (Gil et al., $2007,2008)$. One potential neuronal correlate may be a temporal shift of the onset activity in reinforcement neurons (for review, see Schultz, 2006). The midbrain dopamine neurons in mammals that predict a reward start to respond to the onset of the CS after associative training and stop firing during the onset of the US (for review, see Schultz, 2006). An alternative mechanism is the prolongation of the odor trace after trace conditioning. Associative learning may change the dynamics of the odor-evoked activity and extend the odor trace. Indeed, calcium imaging in dopamine neurons revealed that olfactory conditioning in Drosophila prolonged the response to the trained but not control odor (Riemensperger et al., 2005). Thus, parallel measurements of trace conditioning in behavior and physiology will be important to understand the evolution of odor traces in the brain.

\section{References}

Bathellier B, Buhl DL, Accolla R, Carleton A (2008) Dynamic ensemble odor coding in the mammalian olfactory bulb: sensory information at different timescales. Neuron 57:586-598.

Bellmann D, Richardt A, Freyberger R, Nuwal N, Schwärzel M, Fiala A, Störtkuhl KF (2010) Optogenetically induced olfactory stimulation in Drosophila larvae reveals the neuronal basis of odor-aversion behavior. Front Behav Neurosci 4:27.

Beylin AV, Gandhi CC, Wood GE, Talk AC, Matzel LD, Shors TJ (2001) The role of the hippocampus in trace conditioning: temporal discontinuity or task difficulty? Neurobiol Learn Mem 76:447-461.

Bhandawat V, Olsen SR, Gouwens NW, Schlief ML, Wilson RI (2007) Sensory processing in the Drosophila antennal lobe increases reliability and separability of ensemble odor representations. Nat Neurosci 10:1474-1482.

Carter RM, Hofstotter C, Tsuchiya N, Koch C (2003) Working memory and fear conditioning. Proc Natl Acad Sci U S A 100:1399-1404.

Clark RE, Squire LR (1998) Classical conditioning and brain systems: the role of awareness. Science 280:77-81.

Clark RE, Manns JR, Squire LR (2002) Classical conditioning, awareness, and brain systems. Trends Cogn Sci 6:524-531.

Daly KC, Christensen TA, Lei H, Smith BH, Hildebrand JG (2004) Learning modulates the ensemble representations for odors in primary olfactory networks. Proc Natl Acad Sci U S A 101:10476-10481.

Davis RL (2005) Olfactory memory formation in Drosophila: from molecular to systems neuroscience. Annu Rev Neurosci 28:275-302.

Galán RF, Weidert M, Menzel R, Herz AV, Galizia CG (2006) Sensory memory for odors is encoded in spontaneous correlated activity between olfactory glomeruli. Neural Comput 18:10-25.
Galizia CG, Szyszka P (2008) Olfactory coding in the insect brain: molecular receptive ranges, spatial and temporal coding. Entomol Exp Appl 128: $81-92$.

Galizia CG, Vetter R (2004) Optical methods for analyzing odor-evoked activity in the insect brain. In: Advances in insect sensory neuroscience (Christensen T, ed), pp 349-392. Boca Raton, FL: CRC.

Gerber B, Tanimoto H, Heisenberg M (2004) An engram found? Evaluating the evidence from fruit flies. Curr Opin Neurobiol 14:737-744.

Gervasi N, Tchénio P, Preat T (2010) PKA dynamics in a Drosophila learning center: coincidence detection by rutabaga adenylyl cyclase and spatial regulation by dunce phosphodiesterase. Neuron 65:516-529.

Gil M, De Marco RJ, Menzel R (2007) Learning reward expectations in honeybees. Learn Mem 14:491-496.

Gil M, Menzel R, De Marco RJ (2008) Does an insect's unconditioned response to sucrose reveal expectations of reward? PLoS One 3:e2810.

Guerrieri F, Lachnit H, Gerber B, Giurfa M (2005) Olfactory blocking and odorant similarity in the honeybee. Learn Mem 12:86-95.

Hallem EA, Ho MG, Carlson JR (2004) The molecular basis of odor coding in the Drosophila antenna. Cell 117:965-979.

Ito I, Ong RC, Raman B, Stopfer M (2008) Sparse odor representation and olfactory learning. Nat Neurosci 11:1177-1184.

Jortner RA, Farivar SS, Laurent G (2007) A simple connectivity scheme for sparse coding in an olfactory system. J Neurosci 27:1659-1669.

Kehoe EJ, Ludvig EA, Sutton RS (2009) Magnitude and timing of conditioned responses in delay and trace classical conditioning of the nictitating membrane response of the rabbit (Oryctolagus cuniculus). Behav Neurosci 123:1095-1101.

LaBar KS, Disterhoft JF (1998) Conditioning, awareness, and the hippocampus. Hippocampus 8:620-626.

Larsson MC, Domingos AI, Jones WD, Chiappe ME, Amrein H, Vosshall LB (2004) Or83b encodes a broadly expressed odorant receptor essential for Drosophila olfaction. Neuron 43:703-714.

Masek P, Heisenberg M (2008) Distinct memories of odor intensity and quality in Drosophila. Proc Natl Acad Sci U S A 105:15985-15990.

Masse NY, Turner GC, Jefferis GS (2009) Olfactory information processing in Drosophila. Curr Biol 19:R700-R713.

Mazor O, Laurent G (2005) Transient dynamics versus fixed points in odor representations by locust antennal lobe projection neurons. Neuron 48:661-673.

McEchron MD, Bouwmeester H, Tseng W, Weiss C, Disterhoft JF (1998) Hippocampectomy disrupts auditory trace fear conditioning and contextual fear conditioning in the rat. Hippocampus 8:638-646.

Mishra D, Louis M, Gerber B (2010) Adaptive adjustment of the generalization-discrimination balance in larval Drosophila. J Neurogenet 24:168-175.

Nakai J, Ohkura M, Imoto K (2001) A high signal-to-noise $\mathrm{Ca}^{2+}$ probe composed of a single green fluorescent protein. Nat Biotechnol 19:137-141

Neuser K, Triphan T, Mronz M, Poeck B, Strauss R (2008) Analysis of a spatial orientation memory in Drosophila. Nature 453:1244-1247.

Olsen SR, Bhandawat V, Wilson RI (2007) Excitatory interactions between olfactory processing channels in the Drosophila antennal lobe. Neuron 54:89-103.

Pavlov I (1911) Lectures on conditioned reflexes. In: Transactions of the Society of Russian Physicians, Chap 27, pp 190-191. St. Petersburg: Society of Russian Physicians.

Pelz C, Gerber B, Menzel R (1997) Odorant intensity as a determinant for olfactory conditioning in honeybees: roles in discrimination, overshadowing and memory consolidation. J Exp Biol 200:837-847.

Perez-Orive J, Mazor O, Turner GC, Cassenaer S, Wilson RI, Laurent G (2002) Oscillations and sparsening of odor representations in the mushroom body. Science 297:359-365.

Rescorla RA, Wagner AR (1972) A theory of Pavlovian conditioning: variations in the effectiveness of reinforcement an nonreinforcement. In: Classical conditioning II: current research and theory (Black AH, Prokasy WF, eds), pp 64-99. New York: Appleton-Century-Crofts.

Riemensperger T, Völler T, Stock P, Buchner E, Fiala A (2005) Punishment prediction by dopaminergic neurons in Drosophila. Curr Biol 15: 1953-1960.

Root CM, Masuyama K, Green DS, Enell LE, Nässel DR, Lee CH, Wang JW (2008) A presynaptic gain control mechanism fine-tunes olfactory behavior. Neuron 59:311-321. 
Schaefer AT, Margrie TW (2007) Spatiotemporal representations in the olfactory system. Trends Neurosci 30:92-100.

Schultz W (2006) Behavioral theories and the neurophysiology of reward. Annu Rev Psychol 57:87-115.

Schwaerzel M, Heisenberg M, Zars T (2002) Extinction antagonizes olfactory memory at the subcellular level. Neuron 35:951-960.

Silbering AF, Galizia CG (2007) Processing of odor mixtures in the Drosophila antennal lobe reveals both global inhibition and glomerulus-specific interactions. J Neurosci 27:11966-11977.

Silbering AF, Okada R, Ito K, Galizia CG (2008) Olfactory information processing in the Drosophila antennal lobe: anything goes? J Neurosci 28:13075-13087.

Solomon PR, Groccia-Ellison ME (1996) Classic conditioning in aged rabbits: delay, trace, and long-delay conditioning. Behav Neurosci 110:427435.

Solomon PR, Vander Schaaf ER, Thompson RF, Weisz DJ (1986) Hippocampus and trace conditioning of the rabbit's classically conditioned nictitating membrane response. Behav Neurosci 100:729-744.

Szyszka P, Ditzen M, Galkin A, Galizia CG, Menzel R (2005) Sparsening and temporal sharpening of olfactory representations in the honeybee mushroom bodies. J Neurophysiol 94:3303-3313.

Szyszka P, Galkin A, Menzel R (2008) Associative and non-associative plasticity in kenyon cells of the honeybee mushroom body. Front Syst Neurosci 2:3.

Szyszka P, Demmler C, Oemisch M, Sommer L, Biergans S, Birnbach B, Silbering AF, Galizia CG (2011) Mind the gap: olfactory trace conditioning in honeybees. J Neurosci 31:7229-7239.

Tanimoto H, Heisenberg M, Gerber B (2004) Experimental psychology: event timing turns punishment to reward. Nature 430:983.
Thompson RF, Krupa DJ (1994) Organization of memory traces in the mammalian brain. Annu Rev Neurosci 17:519-549.

Tomchik SM, Davis RL (2009) Dynamics of learning-related cAMP signaling and stimulus integration in the Drosophila olfactory pathway. Neuron 64:510-521.

Tully T, Quinn WG (1985) Classical conditioning and retention in normal and mutant Drosophila melanogaster. J Comp Physiol A Neuroethol Sens Neural Behav Physiol 157:263-277.

Turner GC, Bazhenov M, Laurent G (2008) Olfactory representations by Drosophila mushroom body neurons. J Neurophysiol 99:734-746.

Wang JW, Wong AM, Flores J, Vosshall LB, Axel R (2003) Two-photon calcium imaging reveals an odor-evoked map of activity in the fly brain. Cell 112:271-282.

Wang Y, Guo HF, Pologruto TA, Hannan F, Hakker I, Svoboda K, Zhong Y (2004) Stereotyped odor-evoked activity in the mushroom body of Drosophila revealed by green fluorescent protein-based $\mathrm{Ca}^{2+}$ imaging. J Neurosci 24:6507-6514.

Weiss C, Bouwmeester H, Power JM, Disterhoft JF (1999) Hippocampal lesions prevent trace eyeblink conditioning in the freely moving rat. Behav Brain Res 99:123-132.

Wilson RI, Turner GC, Laurent G (2004) Transformation of olfactory representations in the Drosophila antennal lobe. Science 303:366-370.

Woodruff-Pak DS, Disterhoft JF (2008) Where is the trace in trace conditioning? Trends Neurosci 31:105-112.

Yarali A, Ehser S, Hapil FZ, Huang J, Gerber B (2009) Odour intensity learning in fruit flies. Proc Biol Sci 276:3413-3420.

Yu D, Ponomarev A, Davis RL (2004) Altered representation of the spatial code for odors after olfactory classical conditioning; memory trace formation by synaptic recruitment. Neuron 42:437-449. 